OPEN ACCESS

Edited by:

Sven Krappmann,

University of Erlangen-Nuremberg,

Germany

Reviewed by:

Sean Doyle,

Maynooth University, Ireland

Kai Heimel,

University of Göttingen, Germany

${ }^{*}$ Correspondence:

Robert Marschall

r_mars02@uni-muenster.de; robert.marschall@uni-muenster.de

Specialty section: This article was submitted to Fungi and Their Interactions,

a section of the journa

Frontiers in Microbiology

Received: 24 February 2017

Accepted: 15 May 2017

Published: 29 May 2017

Citation:

Marschall R and Tudzynski P (2017)

The Protein Disulfide Isomerase

of Botrytis cinerea: An ER Protein

Involved in Protein Folding and Redox

Homeostasis Influences NADPH

Oxidase Signaling Processes.

Front. Microbiol. 8:960.

doi: 10.3389/fmicb.2017.00960

\section{The Protein Disulfide Isomerase of Botrytis cinerea: An ER Protein Involved in Protein Folding and Redox Homeostasis Influences NADPH Oxidase Signaling Processes}

\author{
Robert Marschall * and Paul Tudzynski \\ Institut für Biologie und Biotechnologie der Pflanzen, Westfälische Wilhelms-Universität Münster, Münster, Germany
}

Botrytis cinerea is a filamentous plant pathogen, which infects hundreds of plant species; within its lifestyle, the production of reactive oxygen species (ROS) and a balanced redox homeostasis are essential parameters. The pathogen is capable of coping with the plant's oxidative burst and even produces its own ROS to enhance the plant's oxidative burst. Highly conserved NADPH oxidase (Nox) complexes produce the reactive molecules. The membrane-associated complexes regulate a large variety of vegetative and pathogenic processes. Besides their commonly accepted function at the plasma membrane, recent studies reveal that Nox complexes are also active at the membrane of the endoplasmic reticulum. In this study, we identified the essential ER protein BcPdi1 as new interaction partner of the NoxA complex in B. cinerea. Mutants that lack this ER chaperone display overlapping phenotypes to mutants of the NoxA signaling pathway. The protein appears to be involved in all major developmental processes, such as the formation of sclerotia, conidial anastomosis tubes and infection cushions (IC's) and is needed for full virulence. Moreover, expression analyses and reporter gene studies indicate that BcPdi1 affects the redox homeostasis and unfolded protein response (UPR)-related genes. Besides the close association between BcPdi1 and BcNoxA, interaction studies provide evidence that the ER protein might likewise be involved in $\mathrm{Ca}^{2+}$ regulated processes. Finally, we were able to show that the potential key functions of the protein BcPdi1 might be affected by its phosphorylation state.

Keywords: Botrytis, NADPH oxidase, protein folding, ER, virulence, signaling

\section{INTRODUCTION}

Plant pathogenic fungi threaten harvest yields of important economic crops in an increasing manner (Elad et al., 2016). One of the most devastating pathogens, which is responsible for tremendous crops losses of edible fruits and vegetables, is Botrytis cinerea, causative agent of the gray mold disease. The fungus has a necrotrophic lifestyle and benefits from high humidity and moderate temperatures. During vegetative growth, as well as during infection of plant material, various signaling molecules and cascades contribute to the developmental processes. Besides abiotic factors such as light and temperature (Tan and Epton, 1973; Broome et al., 1995; 
Schumacher et al., 2012), internal pathways determine the infection process. Central molecules within pathogenic processes are reactive oxygen species (ROS). ROS are present in all cells that depend on molecular oxygen: they are known to work as signaling molecules at appropriate concentrations, but harmful to macromolecules when present at high concentrations (Beckman and Ames, 1998).

Although plants produce ROS as the first defensive line against pathogen attack, B. cinerea produces its own ROS in highly conserved processes to induce the plant's oxidative burst so that the fungus achieve full pathogenicity (Govrin and Levine, 2000; Heller and Tudzynski, 2011). While ROS result from the activity of the respiratory chain merely as by-products, there are enzymes, such as the NADPH oxidase (Nox) complexes, which actively form superoxide $\left(\mathrm{O}_{2}{ }^{-}\right)$by transporting electrons across the lipid bilayer on to molecular oxygen (Lambeth, 2004; Tudzynski et al., 2012). Nox complexes have been characterized most thoroughly in the mammalian system (reviewed in Laurindo et al., 2014). However, despite the evolutionary distance, there are several homologies between mammalian and fungal Nox complexes. For example, homologs in $B$. cinerea were identified for the catalytic subunit (gp91phox - BcNoxA/B), the regulatory subunit (p67phox - BcNoxR), adaptor proteins (p22phox - BcNoxD) as well as for putative transient members of Nox complexes such as the scaffold protein IQGAP (BcIqg1) (Segmueller et al., 2008; Siegmund et al., 2013, 2015; Marschall and Tudzynski, 2016a). Additionally, mammalian and fungal Nox complexes were shown to have functions in different cellular compartments with a changing complex composition dependent on the respective developmental process (reviewed in Laurindo et al., 2014; Marschall and Tudzynski, 2016b; Marschall et al., 2016a). While the mammalian Nox complexes are involved in cancer (Roy et al., 2015), Alzheimer's disease (Wilkinson and Landreth, 2006) and atherosclerosis (Madamanchi and Runge, 2010), the Nox complexes of $B$. cinerea contribute to a wide range of vegetative and pathogenic processes, such as the formation of infection structures and sporulation (reviewed in Marschall and Tudzynski, 2016b). However, despite major advances made in recent years regarding the composition and function of fungal Nox complexes, there are still many questions about them that remain unanswered.

One of the central questions concerns the link between fungal Nox complexes and calcium $\left(\mathrm{Ca}^{2+}\right)$ signaling processes. Since analyses in plants reveal a tight and recurrent connection between $\mathrm{ROS}$ and $\mathrm{Ca}^{2+}$ signaling pathways (Steinhorst and Kudla, 2014) many studies aim to unravel putative signaling hubs in fungi. Most recently, we were able to show that the protein BcIqg1 interacts with members of ROS and $\mathrm{Ca}^{2+}$ signaling cascades, though it remained unresolved whether the protein contributes to both pathways at the same time or independently (Marschall and Tudzynski, 2016a). Apart from the latter aspects, we have also unraveled the connection between both signaling pathways and the level of redox-mediated processes. Thus, we highlighted that a cytosolic oxidative burst is (1) dependent on both Nox catalytic subunits in B. cinerea and (2) that this oxidative burst appears to rely on elevated $\mathrm{Ca}^{2+}$ levels. Moreover, we elucidated that increasing $\mathrm{Ca}^{2+}$ concentrations promote a slight oxidative burst inside the endoplasmic reticulum (ER), mediated by the catalytic subunit BcNoxA (Marschall et al., 2016b). The ER is commonly accepted as a $\mathrm{Ca}^{2+}$ storage compartment (Koch, 1990), and previous studies established that both Nox complexes have distinct functions at the ER membrane (Siegmund et al., 2015; Marschall et al., 2016a). It is reasonable to suggest therefore that the ER plus its associated proteins may be a central location for the transmission of signals, and for the physical interaction of proteins belonging to ROS and $\mathrm{Ca}^{2+}$ signaling cascades.

The main function of the ER is the processing of (especially secretory) proteins by the oxidative protein folding machinery. Primarily responsible for the reduction, isomerization or oxidation of proteins within this process is the protein disulfide isomerase (PDI). In its oxidized form, PDI is able to function as a posttranslational modifying chaperone, which facilitates correct protein folding and catalyzes disulfide exchange reactions. During this process PDI is reduced and has to be regenerated by the oxidoreductin 1 (Ero1) (Kersteen and Raines, 2003). PDIs are highly conserved proteins that are involved in various developmental processes. Whereas in plants they are known to affect the development of endosperm (Li and Larkins, 1996) and gene expression in chloroplasts (Kim and Mayfield, 1997), in mammalian systems they were shown to influence intracellular $\mathrm{Ca}^{2+}$ concentrations (Corbett and Michalak, 2000; Lucero et al., 1998), apoptotic processes (Ko et al., 2002) and stress signaling (Sullivan et al., 2003). In mammals, however, the proteins are associated or directly interact with several important proteins of essential signaling pathways, such as calreticulin $\left(\mathrm{Ca}^{2+}\right.$ signaling, Baksh et al., 1995) or members of the Nox complexes (Janiszewski et al., 2005; Laurindo et al., 2008; Fernandes et al., 2009; de A Paes et al., 2011).

In fungi, studies on PDIs showed that, due to its folding properties, the protein is involved in the secretory pathway (Ngiam et al., 2000), as well as being activated by exposure to stress (Saloheimo et al., 1999). Therefore, the protein clearly belongs to a large set of proteins, which are upregulated under ER stress and during the unfolded protein response (UPR) across all species boundaries (Haefliger et al., 2011; Perri et al., 2016). Moreover, the protein is essential for viability in Saccharomyces cerevisiae (Farquhar et al., 1991). However, information about interaction partners of PDI and its involvement in existing signaling networks is lacking.

Here, we report the occurrence of physical interactions between members of different signaling pathways. The close association of the PDI BcPdil to $\mathrm{Ca}^{2+}$ and ROS signaling cascades was determined by studying $\Delta b c p d i 1$ mutants in comparison to deletion mutants of the Nox and $\mathrm{Ca}^{2+}$ signaling pathways. Many overlapping phenotypes in both vegetative and pathogenic processes were observed. The protein BcPdil appears to be essential for all major developmental processes, such as the formation of sclerotia, conidial anastomosis tubes (CATs) and infection cushions (IC's), as well as for full virulence. Finally, we were able to show that the potential key function of the protein BcPdil may be affected by its phosphorylation state, which likewise is necessary for the maintenance of the redox homeostasis and the transmission of signals. 


\section{MATERIALS AND METHODS}

\section{Cultivation of Botrytis cinerea}

Botrytis cinerea Pers.:Fr. [Botryotinia fuckeliana (de Bary)Whetzel] B05.10 was collected from Vitis vinifera (Buettner et al., 1994) and was used in this study as basis strain and control in all experiments. Further strains are listed in Supplementary Table S1.

For cultivation synthetic complete medium (CM) (Pontecorvo et al., 1953) was used. For transformation, the strains were grown on PDAB medium (Potato dextrose agar [Sigma-Aldrich Chemie, Steinheim, Germany] supplemented with $100 \mathrm{~g} / \mathrm{l}$ homogenized leaves of French beans (Phaseolus vulgaris). Cultures were grown for $6-8$ days at $20^{\circ} \mathrm{C}$ under light conditions (12 h light/12 h darkness, full spectrum light) to obtain conidia. Sclerotia were induced by incubating the strains for 3 weeks at $20^{\circ} \mathrm{C}$ in darkness. For DNA preparation, the strains were grown for 3-4 days at $18^{\circ} \mathrm{C}$ on $\mathrm{CM}$ agar overlayed with Cellophane. For stress experiments, the $\mathrm{CM}$ was supplemented with agents inducing osmotic, oxidative, cell wall, membrane or ionic stress. Minimal medium was prepared after Czapek Dox ( $20 \mathrm{~g} / \mathrm{l}$ sucrose, $3 \mathrm{~g} / \mathrm{l} \mathrm{NaNO}_{3}, 1 \mathrm{~g} / \mathrm{l} \mathrm{K} \mathrm{HPO}_{4}, 0.5 \mathrm{gl} \mathrm{KCl}, 0.01 \mathrm{~g} / \mathrm{l} \mathrm{FeSO}{ }_{4} \times 7 \mathrm{H}_{2} \mathrm{O}$, $0.5 \mathrm{~g} / \mathrm{l} \mathrm{MgSO}_{4} \times 7 \mathrm{H}_{2} \mathrm{O}, \mathrm{pH} 5.2$ ).

\section{Generation of Mutant Strains}

For the generation of deletion and complementation constructs, the yeast homologous recombination system was used (Colot et al., 2006; Schumacher, 2012). For generating the deletion cassette of $b c p d i 1$, the $5^{\prime} / 3^{\prime}$ flank were amplified with the primer $1 / 2$ and 3/4 (Supplementary Table S2) as well as the resistance cassette with the primer pair 16/17. Recombinational cloning was done with S. cerevisiae FY834 and the vector backbone of pRS426 (EcoRI/XhoI). For complementation, bcpdi1 was expressed under its native promotor (primer 10/15) as well as under the constitutive oliC promotor of Aspergillus nidulans (primer 9/10). The constructs for introducing mutated phosphorylation sites were amplified using the primer $9 / 12$ and 10/11 ( $\Delta b c p d i 1-\mathrm{C}$ M1), the primer $9 / 14$ and 10/13 ( $\Delta b c p d i 1-\mathrm{C}-\mathrm{M} 2), 9 / 12,11 / 14$ and 10/13 ( $\Delta b c p d i 1-\mathrm{C}-\mathrm{M} 1 / 2), 10 / 29$ and 9/28 ( $\Delta b c p d i 1-\mathrm{C}-\mathrm{DA})$. For the generation of PDI in loco complementation constructs that were fused to the genetically encoded biosensor roGFP2, six different fragments were generated by the primer pairs $1 / 11$, 12/32, 33/34, 35/36, 37/38, 39/4 (PDI_phospho_null) or 1/28, 29/30, 31/32, 33/34, 35/36, 37/4 (PDI_phospho_mimic).

After transforming S. cerevisiae FY834, positive transformants were selected on SD medium lacking uracil since the strain is auxotrophic for this amino acid. Total DNA was isolated and transformed in Escherichia coli. Re-isolation was done by the Nucleo spin ${ }^{\circledR}$ plasmid easypure kit (Macherey-Nagel, Düren, Germany) and correct assembly was tested by sequencing.

Botrytis cinerea was transformed with $40-60 \mu \mathrm{g}$ of plasmid DNA as described previously (Gronover et al., 2001). Selection was done via hygromycin $(70 \mu \mathrm{g} / \mathrm{ml}$ of hygromycin B (Invitrogen, San Diego, CA, United States) or nourseothricin $(50 \mu \mathrm{g} / \mathrm{ml}$ of nourseothricin (Werner-Bioagents, Jena, Germany). Positive transformants were purified by single spore isolation: Conidia were spread on the appropriate selective medium; afterward, germinated conidia were picked and cultivated on new selective plates. Genomic DNA isolation was performed according to Cenis (Cenis, 1992). Correct and ectopic integrations were checked by diagnostic PCR and Southern blot (primer 18-27).

\section{Growth and Pathogenicity Assays}

For characterization of the different mutant strains various growth, differentiation and pathogenicity assays were performed. For testing defects in pathogenicity, French bean plants (Phaseolus vulgaris L.) were infected according to Klimpel et al. (2002) with agar plugs or conidia from freshly sporulated PDAB plates. Germination on glass surfaces was tested as described by Doehlemann et al. (2006). Penetration ability was checked on onion epidermal layers after inoculation with washed conidia. Before microscopy, the hyphae growing on top of the layers were stained with lactophenol blue. For observation of ICs, the epidermal layers were inoculated with agar plugs of mycelium and incubated overnight (Siegmund et al., 2015). For visualization of CATs media and strains were prepared following Siegmund et al. (2015).

\section{Northern Blot Analysis}

For Northern blot analyses, strains were grown on CM overlayed with Cellophane for at least 3 days. The mycelium was harvested, lyophilized and the RNA was isolated via the Trizol procedure (Invitrogen, Groningen, The Netherlands). Samples of $20 \mu \mathrm{g}$ were loaded to an agarose gel and transferred to Hybond-N+ membranes. Northern blot hybridizations were done according to the method of Church and Gilbert (1984). The different probes used for expression analysis were generated by PCR with the primer pairs 40/41 (Hac1) and 42/43 (BiP/Kar2).

\section{Epifluorescence Microscopy}

Light microscopy imaging was performed using the Axio Imager 2 and the Axiovert (Zeiss, Jena, Germany). Visualization of ICs was done with the $20 \times$ objective lens, while germinated conidia were analyzed with $40 \times$ or $63 \times$ magnification. ER staining was accomplished using the ER-Tracker ${ }^{\mathrm{TM}}$ Blue-White DPX (Life Technologies, Germany) in McIlvaine standard buffer (McIlvaine, 1921). The samples were observed via the filter set 49 DAPI shift free (excitation G 365, beam splitter FT 395, emission BP 445/50). GFP fluorescence was detected with filter set 38 (excitation BP 470/40, beam splitter FT 495, emission BP 525/50). Images were captured with a Zeiss AxioCamMRm camera and further processed using the Axiovision Rel 4.8 software package.

\section{Confocal Laser Scanning Microscopy (CLSM) Imaging and Ratiometric Analysis}

roGFP2 measurements were done using an inverted microscope (Leica DMIRE2) equipped with a Leica TCS SP2 scan head (Leica Microsystems, Wetzlar, Germany). Conidia were prepared as described previously (Heller et al., 2012). Results were obtained by using the excitation wavelengths 395 (first track) and 488 
(second track) as well as a 505-530 bandpass filter for collecting images. Z-stacks were displayed as average projections via the CLSM software. Further evaluation was done with the Image J program (v.1.44f $\mathrm{f}^{1}$ ) as it was shown before (Marschall et al., 2016b).

\section{Interaction Studies}

Protein-Protein interactions were investigated by a splitubiquitin based yeast-two-hybrid system (Dual System Biotech). The proteins of interest were fused to the C- or N-terminal half of ubiquitin. Upon interaction, ubiquitin congregates and the transcription factor controlling reporter genes (histidine and adenine - see also manual Dual Systems Biotech, Schlieren, Switzerland) can be released. Proteins without transmembrane regions are additionally fused to a transmembrane domain of the oligosaccharyltransferase subunit $4(\mathrm{Ost} 4)$ of $S$. cerevisiae for anchoring the proteins at the membrane. Interaction vectors (prey/bait) were generated according to the manufacturer's protocol. Both vectors $(\mathrm{pPR} 3 \mathrm{~N}=$ prey; $\mathrm{pDHB} 1=$ bait $)$ were restricted with SfiI and ligated with likewise restricted PCR products (for details see Supplementary Table S2). The ligated products were transformed in E. coli and selected with the appropriate selection marker.

For interaction assays, prey and bait were transformed in S. cerevisiae NMY51 according to the manufacturer's protocol (DUALSystems Biotech) with a modified version of the lithiumacetate method (Gietz and Woods, 2002). Yeast cells were selected on SD-agar lacking the appropriate amino acids (' $l e u^{-} \operatorname{trp}$ as control of vector transformation; ${ }^{-} l e u^{-}{ }^{-} r p^{-}$his ${ }^{-}$ade as test for positive interaction). For drop tests, established colonies were grown overnight in SD medium lacking leucine and tryptophan. $\mathrm{OD}_{600}$ was adjusted to 1 and cells were pelleted. The medium was removed and starvation was induced by the addition of sorbitol $(1 \mathrm{M})$ : incubation then took place for at least $5 \mathrm{~h}$ at $30^{\circ} \mathrm{C}$ and $200 \mathrm{rpm}$ on a shaker. Subsequently samples were diluted up to 1:1000 and $10 \mu \mathrm{l}$ of each dilution were dropped on selective medium ( $\mathrm{SD}^{-}$leu ${ }^{-}$trp $^{-}$his ${ }^{-}$ade $+5 \mathrm{mM} 3-\mathrm{AT}+80 \mathrm{mg} / \mathrm{l}$ $\mathrm{X}-\mathrm{Gal})$ and control plates $\left(\mathrm{SD}^{-} l e u^{-} \operatorname{trp}\right)$. Plates were incubated for 3-4 days at $30^{\circ} \mathrm{C}$.

For co-IP experiments, proteins of interest were transformed into the wild type strain. The expression is controlled by the constitutive oliC promotor (A. nidulans) and reporter genes were fused to the $\mathrm{N}$ - or $\mathrm{C}$ terminus of the respective protein. Fusion proteins were generated by the yeast recombination system. Whereas bcnoxA was cloned into the vector pNAH_OCT (restricted with $\mathrm{NcoI}$ ) containing mcherry as reporter gene, bcpdi1 was cloned into the vector pNDN_OGG (restricted with NcoI) with $g f p$ as fluorescent marker.

After transformation, the strain expressing both constructs was purified by single spore isolation and grown on CM medium with Cellophane overlay for 3-4 days. The mycelium was harvested, lyophilized and proteins were isolated by grinding and applying disruption buffer $[20 \mathrm{mM}$ Tris/HCL $\mathrm{pH}$ 8, $150 \mathrm{mM} \mathrm{NaCl}, 0.05 \%$ Triton X-100 supplemented with $10 \mu \mathrm{l}$ Phosphatase inhibitor $1 / 2$ and $10 \mu l$ of Protease inhibitor

\footnotetext{
${ }^{1}$ http://rsb.info.nih.gov/ij/
}

(Sigma-Aldrich, Steinheim, Germany)]. For the NoxA_mcherry control, the extract was stored until Western blotting. The protein extract of the PDI_GFP control as well as of the strain expressing both constructs was loaded to $\mu \mathrm{MACS}$ GFP beads/columns and purified according to the instruction manual of the $\mu$ MACS GFP tagged protein isolation kit (Milteny Biotech Bergisch Gladbach, Germany). After Western blotting, detection was accomplished using the anti-gfp antibody (Milteny Biotech, Bergisch Gladbach, Germany). For mcherry detection, the anti-mcherry antibody (Thermo Fisher Scientific, Schwerte, Germany) was used as primary antibody and for the secondary antibody the donkey-anti-rabbit antibody (Thermo Fisher Scientific, Schwerte, Germany) was used. Visualization was conducted using the enhanced chemi-luminescence (ECL) detection (Bio-Rad, Munich, Germany).

\section{Database Resources}

Nucleotide and protein sequences of B. cinerea strain B05.10 were obtained by the database Ensembl ${ }^{2}$. For the analysis of the sequences, different programs were used: Signal peptides were predicted by SOSUIsignal (Gomi et al., 2000), subcellular localization patterns of proteins were predicted by ProtComp v.9. $0^{3}$, transmembrane regions were predicted by using the programs TMHMM ${ }^{4}$ and SACS MEMSAT2 (Jones et al., 1994). Phosphorylation sites were predicted by KinasePhos (Huang et al., 2005).

\section{RESULTS}

\section{Functional Analysis of the Protein Disulfide Isomerase BcPdi1}

Since our studies had revealed that the NoxA complex has a distinct function inside the ER, putative targets/partners were investigated. An essential ER protein, which in mammalian systems is associated with subunits of the Nox complex (Janiszewski et al., 2005; Laurindo et al., 2008; Fernandes et al., 2009; de A Paes et al., 2011) is the PDI. Furthermore, the putative homolog to the mammalian protein was identified as a putative interaction partner of BcNoxA in a cDNA library screen (Siegmund and Tudzynski, unpublished). Blastp analysis and multiple sequence alignments using Clustal $\Omega$ (Sievers et al., 2011) displayed a query coverage of $85 \%$ as well as a sequence identity of $37 \%$ by comparing the mammalian PDI with the fungal protein (Bcin06g05730). According to the Ensemble Botrytis genome database ${ }^{5}$ the ORF consists of 1596 bp encoding a protein of 531 aa. Bioinformatic analyses revealed that the protein (BcPdil) probably contains two transmembrane domains with a small cytosolic loop (Supplementary Figure S1A), with both ends located inside the ER. Its two catalytic domains (CXXC-motif) are predicted to be located in the cytosolic

\footnotetext{
${ }^{2} \mathrm{http} / / /$ fungi.ensembl.org/Botrytis_cinerea/Info/Index

${ }^{3} \mathrm{http} / / / \mathrm{www} \cdot$ softberry.com/berry.phtml?topic=protcompan\&group=programs\& subgroup $=$ proloc

${ }^{4} \mathrm{http}: / /$ www.cbs.dtu.dk/services/TMHMM/

${ }^{5} \mathrm{http}: / /$ fungi.ensembl.org/Botrytis_cinerea/Info/Index
} 
loop (1 in Supplementary Figure S1A) and inside the ER, respectively (4 in Supplementary Figure S1A). Moreover, two cytosolic phosphorylation sites are predicted $(2 / 3$ in Supplementary Figure S1A). Interestingly, the first putative phosphorylation site is highly conserved among different fungal species (Supplementary Figure S1A) while the second one is poorly conserved.

For functional characterization, deletion mutants of bcpdi1 were generated (Supplementary Figure S1B). Transformants were checked via diagnostic PCR (Supplementary Figure S1B) and Southern blot analysis (Supplementary Figure S1C for details see Supplementary Tables S1, S2). Since all mutants displayed an identical phenotype, the results of the mutant $\Delta b c p d i 1 \_\mathrm{T} 2$ are presented. In order to elucidate the role of the predicted cytosolic phosphorylation sites of the protein, mutants with mutated phosphorylation sites were generated. The key amino acids were replaced by an alanine to mimic the constitutively dephosphorylated state (phospho null) (BcPdi1_M1 = T65A; BcPdi1_M2 = Y208A). Moreover, the putative first phosphorylation site was transferred into its constitutively phosphorylated form (phospho mimic) by replacing threonine by glutamic acid (T65E).

\section{BcPdi1 Is Involved in Oxidative and Osmotic Stress Resistance}

To check whether BcPdil is involved in stress response pathways, the radial growth was determined on $\mathrm{CM}$ containing various stress agents.

The radial growth of the $\Delta b c p d i 1$ mutant on $\mathrm{CM}$ was reduced by $20 \%$ in comparison to the wild type. Whereas the complemented strain, $\Delta b c p d i 1-\mathrm{C}-\mathrm{M} 2$ and $\Delta b c p d i 1-\mathrm{C}-\mathrm{DA}$ grew as well as the wild type, the colony diameters of $\Delta b c p d i 1-\mathrm{C}-\mathrm{M} 1$ (87.5\%) and $\Delta b c p d i 1-\mathrm{C}-\mathrm{M} 1 / 2(83.5 \%)$ were slightly reduced.

The deletion of $b c p d i 1$ led to a higher sensitivity of the mutants to oxidative and osmotic stress. The addition of $10 \mathrm{mM} \mathrm{H}_{2} \mathrm{O}_{2}$ and on $1 \mathrm{M} \mathrm{NaCl}$ was sufficient to reduce the rate of radial growth of $\Delta b c p d i 1$ significantly, but the addition of menadione had no effect. While the complemented strain displayed wild type-like growth on all selective media, the mutation of the first predicted phosphorylation site correlated with the deletion mutant's phenotype. The mutation of the second putative phosphorylation site had no effect (Figure 1). The 'phospho mimic' mutants mostly resembled the growth behavior of the wild type. However, on $\mathrm{NaCl}$-supplemented medium the mutant grew much better than all other strains. In contrast to oxidative and osmotic stress, the addition of Calcofluor White $(2 \mathrm{mg} / \mathrm{ml})$ as a cell wall stressor, or SDS $(0.02 \%)$ as a membrane stressor did not differentially influence the radial growth of the mutants compared to the wild type.

\section{BcPdi1 Affects Production of Conidiospores and Virulence}

Botrytis cinerea is able to infect its host from spore- or myceliummediated penetration processes. Whereas appressoria are formed after the short germination of a fungal spore, so-called ICs are mycelium-derived penetration structures that facilitate the breakthrough of the plant cell wall (Armentrout and Downer, 1987).

The deletion of bcpdil attenuated the spore-mediated infection of bean leaves, but the way the mutant formed appressoria appeared as in the wild type (Figures 2A,C). For this study, spores were inoculated on onion epidermal layers and fungal material was stained with lactophenol blue after $16 \mathrm{~h}$, allowing discrimination between the stained surface hyphae and those left unstained after penetration of the host. The mutants were able to penetrate the plant's surface but the subsequent colonization was severely inhibited (Figure 2A). A similar phenotype was recorded for the strains $\Delta b c p d i 1-\mathrm{C}-\mathrm{M} 1$ as well as $\Delta$ bcpdi1-C-M1/2. In marked contrast, the complemented strain $(\Delta b c p d i 1-\mathrm{C})$ and $\Delta b c p d i 1-\mathrm{C}-\mathrm{M} 2$ appeared to infect in the manner of the wild type.

All strains that displayed a retarded colonization of plant tissue produced significantly fewer spores than the wild type and the strains that restored the wild type phenotype (Figure 2B). However, since all plants were infected with the same concentration of conidiospores, there is no causal correlation between reduced production of conidia and impaired virulence.

When studying the mycelium-mediated infection process of B. cinerea similar results were obtained as previously for the spore-mediated infection. In contrast to the wild type and the strains $\Delta b c p d i 1-\mathrm{C}$ and $\Delta b c p d i 1-\mathrm{C}-\mathrm{M} 2$, the deletion mutant was impaired in its ability to achieve mycelium-mediated penetration as well as in the colonization stage (Figure 3A). Primary lesions were not visible until the 3 rd day of infection. Similar results were obtained for $\Delta b c p d i 1-\mathrm{C}-\mathrm{M} 1$ and $\Delta b c p d i 1-\mathrm{C}-\mathrm{M} 1 / 2$.

The defects in infecting plant tissue may be due to the impaired production of ICs. All strains that were unaffected in the mycelium-mediated infection process were still able to produce ICs. In contrast, the mutant strains $\Delta b c p d i 1, \Delta b c p d i 1-\mathrm{C}-\mathrm{M} 1$, and $\Delta$ bcpdi1-C-M1/2 were unable to produce wild type-like ICs, but instead produced twisted hyphae which were not targeted toward one infection site as is normal for the wild type (Figure 3B).

\section{BcPdi1 Influences the Formation of Hyphal Fusions and Sclerotia}

Since it has been shown previously that members of the NoxA complex as well as putative interaction partners are impaired in the formation of CATs (Roca et al., 2012; Siegmund et al., 2015; Marschall and Tudzynski, 2016b), the different PDI strains were investigated with respect to this differentiation process. These specialized hyphae are formed to exchange cellular material between two hyphae of the same organism. Whereas the wild type, as well as the strain $\Delta b c p d i 1-\mathrm{C}$, were still capable of forming CATs, the deletion mutant and the strains $\Delta b c p d i 1-\mathrm{C}$ M1, $\Delta b c p d i 1-\mathrm{C}-\mathrm{M} 1 / 2$, and $\Delta b c p d i 1-\mathrm{C}-\mathrm{DA}$ were impaired in the formation of hyphal fusions. The mutant $\Delta b c p d i 1-\mathrm{C}-\mathrm{M} 2$ was still able to form the hyphal structures, but produced markedly less CATs than the wild type (Figure 2D).

Apart from hyphal fusions, BcPdil also affects the production of perennial survival structures called sclerotia. These structures, which are produced under constant darkness, were formed by the 


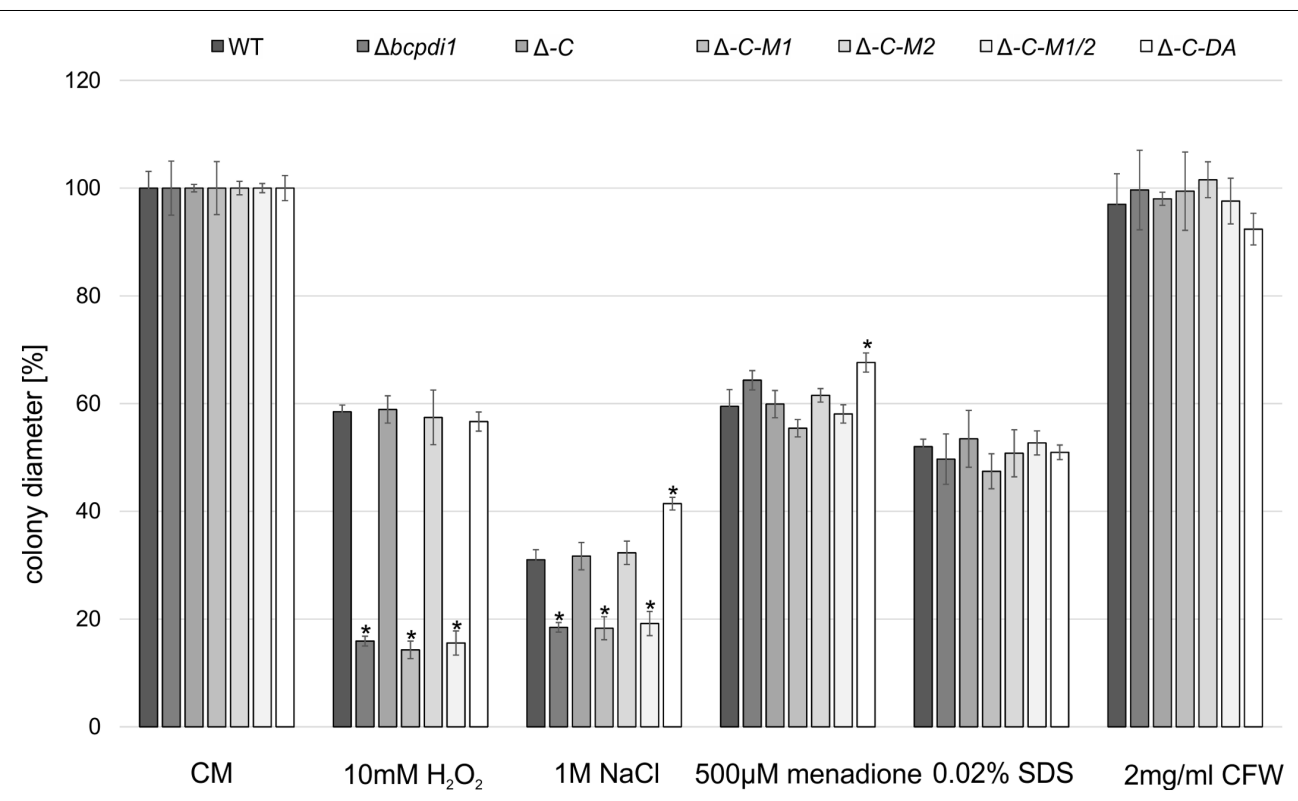

FIGURE 1 | BcPdi1 mediates stress resistance against oxidative and osmotic stress. Agar plugs of 3-days-old CM plates were incubated on CM plates supplemented with $10 \mathrm{mM} \mathrm{H} \mathrm{O}_{2}, 1 \mathrm{M} \mathrm{NaCl}, 500 \mu \mathrm{M}$ menadione, $0.02 \%$ SDS or $2 \mathrm{mg} / \mathrm{ml}$ Calcofluor White. Colony diameters were measured and compared to the wild type (here after 3 days). Replicates showed similar results. Mean values and standard deviations were calculated from three independent experiments. Asterisks indicate significant differences to the wild-type control in every condition ( $t$-test, $P<0.01$ ).

wild type and the complemented strain $\Delta$ bcpdi1-C (Figure 3C). All other strains were defective in the production of sclerotia. Surprisingly, the mutant strain $\Delta b c p d i 1-\mathrm{C}-\mathrm{M} 2$ also failed to complement the defect in sclerotial development, although this mutant behave as the wild type in all other respects.

To summarize, BcPdil affects important vegetative and pathogenic processes, such as sclerotial formation, hyphal fusions and penetration structures. Essential for these developmental processes is the cytosolic region of $\mathrm{BcPdil}$ containing the two putative phosphorylation sites as well as one catalytically active site. Whereas the second predicted phosphorylation site seems to have an exclusive effect on the production of sclerotia, the first site seems to be essential for all essential protein functions.

\section{BcPdi1 Localizes to the ER}

To study the localization pattern of BcPdil, a GFP fusion construct was generated under the control of the native promoter (1 kb upstream of the ORF) and the constitutive oliC promoter. Both were transformed into the deletion mutant's background. The wild type phenotype was fully restored by both complementation constructs (Data not shown). Additionally, in both cases the target protein localized to identical structures. The strain BcPdi1-C was used since the fluorescence of the protein under the constitutive oliC promoter was much brighter for localization studies and later experiments. Apart from re-integrating bcpdil into its wild type version, GFP-fusion constructs with the mutated alleles ( $\Delta$ bcpdi1-C-M1, $\Delta$ bcpdi1-CM2, $\Delta$ bcpdi1-C-M1/2, $\Delta$ bcpdi1-C-DA-M1) were generated.

BcPdil localized to filamentous structures (Figure 4) belonging to the ER (verified by co-staining with ER tracker) and was additionally found in the nuclear envelope (verified by co-staining with the DNA dye Hoechst 33342 - Data not shown). Although the mutation of the second putative phosphorylation site (M2) into its phospho null form had no influence on the localization, the pattern changed upon mutation of M1. BcPdi1_M1 was detected in a faint network of filamentous structures belonging to the ER, but was hardly visible in the nuclear envelope. Moreover, the proteins seemed to adhere as aggregates that were detected throughout the hyphae. Similar results were obtained for the BcPdi1_GFP fusion construct containing both mutated phosphorylation sites.

The localization pattern of the phospho mimic mutants differed from all other observed PDI-constructs. The fusion constructs localized to filamentous structures, which show high similarity to mitochondria. Co-localization with the mitoIDred mito tracker as well as with the ER tracker revealed that those structures are overlapping with ER and mitochondrial structures (Figure 4).

In summary, we have established that BcPdil is indeed an ER protein that is located within the nuclear envelope and filamentous structures. Mutations of the first predicted cytosolic phosphorylation site of the protein directly influenced the distribution of the protein within hyphae, a most unexpected result.

\section{BcPdi1 Affects the Cytoplasmic Redox State}

To elucidate the role of BcPdil both inside and outside the ER, studies with the genetically encoded biosensor roGFP2 were performed. The modified version of the reporter gene 

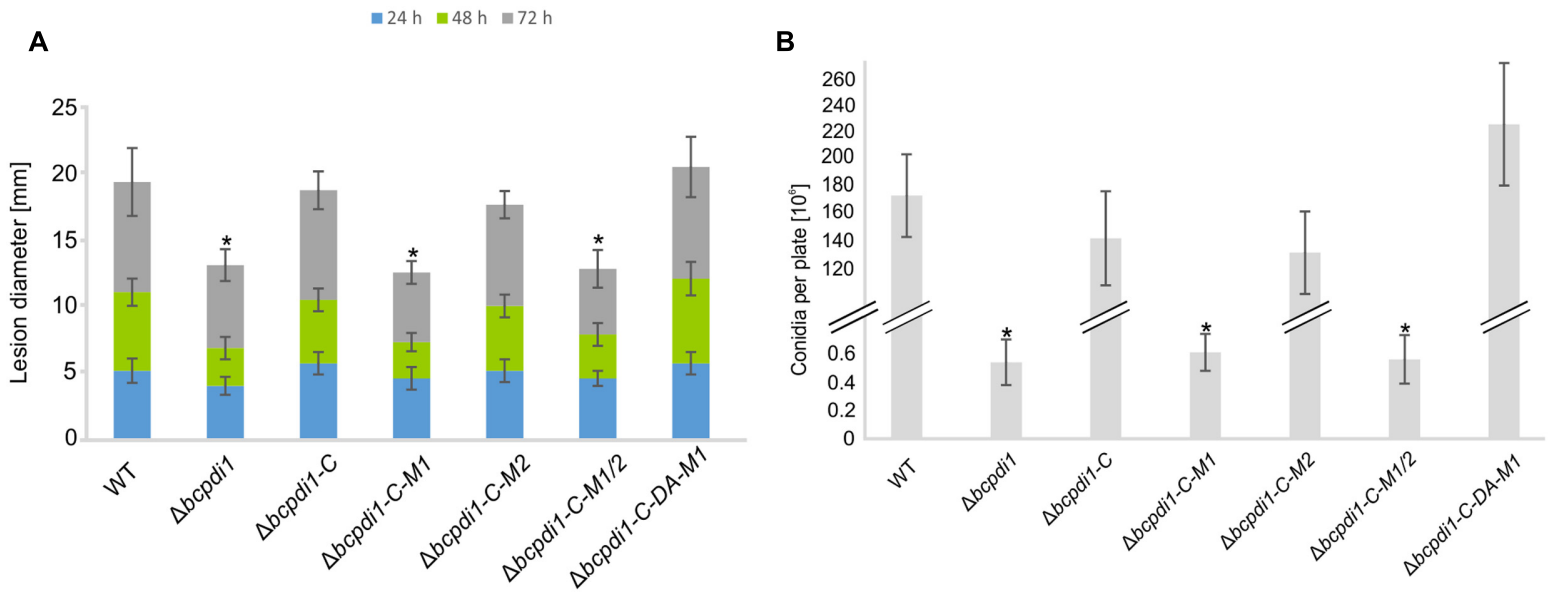

C
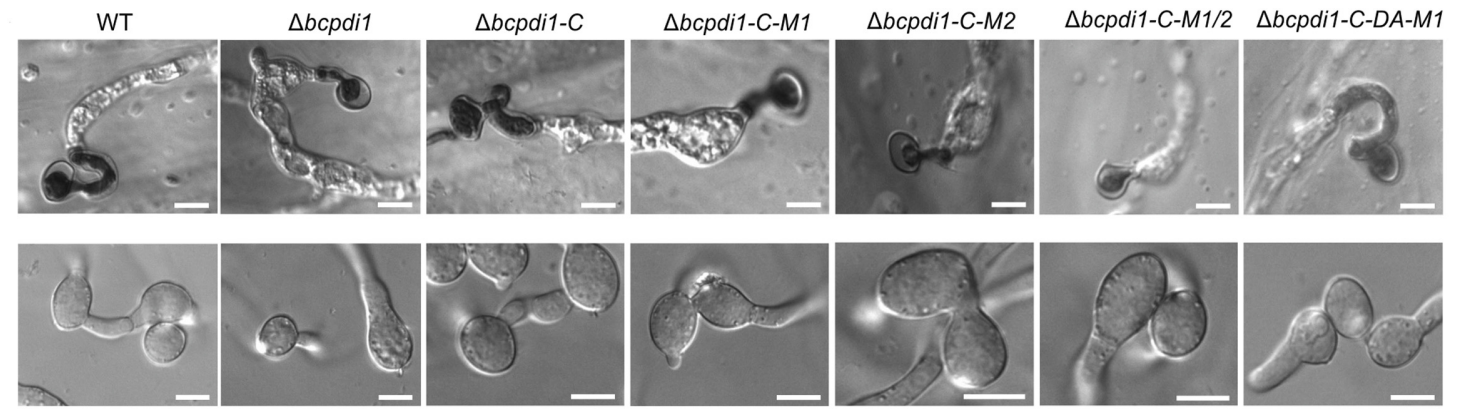

FIGURE 2 | The deletion of bcpdi1 impacts spore mediated infection, CAT and conidia formation. (A) Primary bean leaves were inoculated with conidia $\left(10^{5}\right)$. Lesion diameters were measured each day and evaluated statistically. Significant differences were indicated by asterisks ( $t$-test, $P<0.01)$. Replicates showed similar results. (B) Mutant strains of bcpdi1 are impaired in the production of conidiospores. Agar plugs were incubated on CM plates for at least 3 weeks. Conidia were washed down and counted. Results of five biological replicates were similar. Mean values and standard deviations were calculated from five independent experiments. Significant differences to the wild type control were indicated by asterisks ( $t$-test, $P<0.01$ ). (C) The deletion of bcpdi1 leads to defects in hyphal fusions. Conidia were plated on Vogel's minimal medium and incubated for $18 \mathrm{~h}$. For each strain 300 conidia were investigated concerning their fusion ability. (D) The penetration ability is unaltered in the mutants of bcpdi1. Onion epidermal layers were inoculated with conidia. Just before microscopy, lactophenol blue staining was performed to visualize the fungal material on the surface of the onion layers.

was suitable for the visualization of the glutathione pool, which mirrors the current redox state of the monitored compartment (Schwarzlaender et al., 2008; Heller et al., 2012). These studies aimed to establish, whether BcPdil affects the cytosolic redox state and, therefore, is involved in the maintenance of the intracellular redox balance. Under non-inducing conditions, the phospho null version ( $\left.\triangle b c p d i 1-\mathrm{C}-\mathrm{M} 1 \_r o G F P 2\right)$ appeared like the wild type and the deletion mutant (data not shown) whereas the phospho mimic version ( $\left.\triangle b c p d i 1-C-D A \_r o G F P 2\right)$ exhibited an elevated $395 / 488$ ratio (Figure 5A top).

Since previous studies with mutants of both Nox complexes revealed that $\mathrm{CaCl}_{2}$ is sufficient to induce an oxidative burst inside the cytosol, the mutants ( $\triangle b c p d i 1-\mathrm{C}-\mathrm{M} 1$ _roGFP2; $\triangle b c$ di1-C-DA_roGFP2) were also tested under those conditions $(50 \mathrm{mM} \mathrm{CaCl})_{2}$. Surprisingly, both patterns of the mutants did not resemble the wild type control. In the wild type, the addition of $\mathrm{CaCl}_{2}$ induced a massive oxidative burst, changing the $395 / 488$ ratio from 0.3 to 1.4 . In comparison, the $395 / 488$ ratio of $\Delta b c p d i 1-\mathrm{C}-\mathrm{M} 1 \_$roGFP2 remained unaffected as the deletion mutant $\Delta b c p d i 1$ (data not included in the figure, identical curve), and the $395 / 488$ ratio of the phospho mimic version ( $\Delta$ bcpdi1-CDA_roGFP2) was slightly shifted to a more oxidized state than the wild type (Figure 5A below). The induction via $\mathrm{CaCl}_{2}$ led only to minor changes of the $395 / 488$ ratio.

To elucidate, why the basic $395 / 488$ ratio of $\Delta b c p d i 1-\mathrm{C}-$ DA_roGFP2 increased in comparison to the wild type, we did expression studies with known marker genes of the UPR. Since previous studies in plant cells revealed that changes in the redox state are due to the activity of the UPR machinery (Merksamer et al., 2008) we analyzed the $b c p d i 1$ mutants expressing roGFP2 with respect to UPR activity. We used the putative $B$. cinerea homologs of the genes BiP/Kar2 (Bcin13g00960) and Hac1 (Bcin08g06730). Whereas $\mathrm{BiP} / \mathrm{Kar} 2$ is a chaperone responsible for the correct folding of proteins (Jung et al., 2013), the transcriptional activator $\mathrm{Hacl}$ binds to UPR-responsive elements to increase the amount of ER-resident proteins required for the folding machinery as well as components of the secretory pathway (Mori et al., 1992, 1998). Both were analyzed in the wild type in comparison to $\Delta b c p d i 1-\mathrm{C}-\mathrm{M} 1 \_$roGFP2 and $\Delta b c p d i 1$-CDA_roGFP2. The expression of the $\mathrm{BiP} / \mathrm{Kar} 2$ homolog appeared 


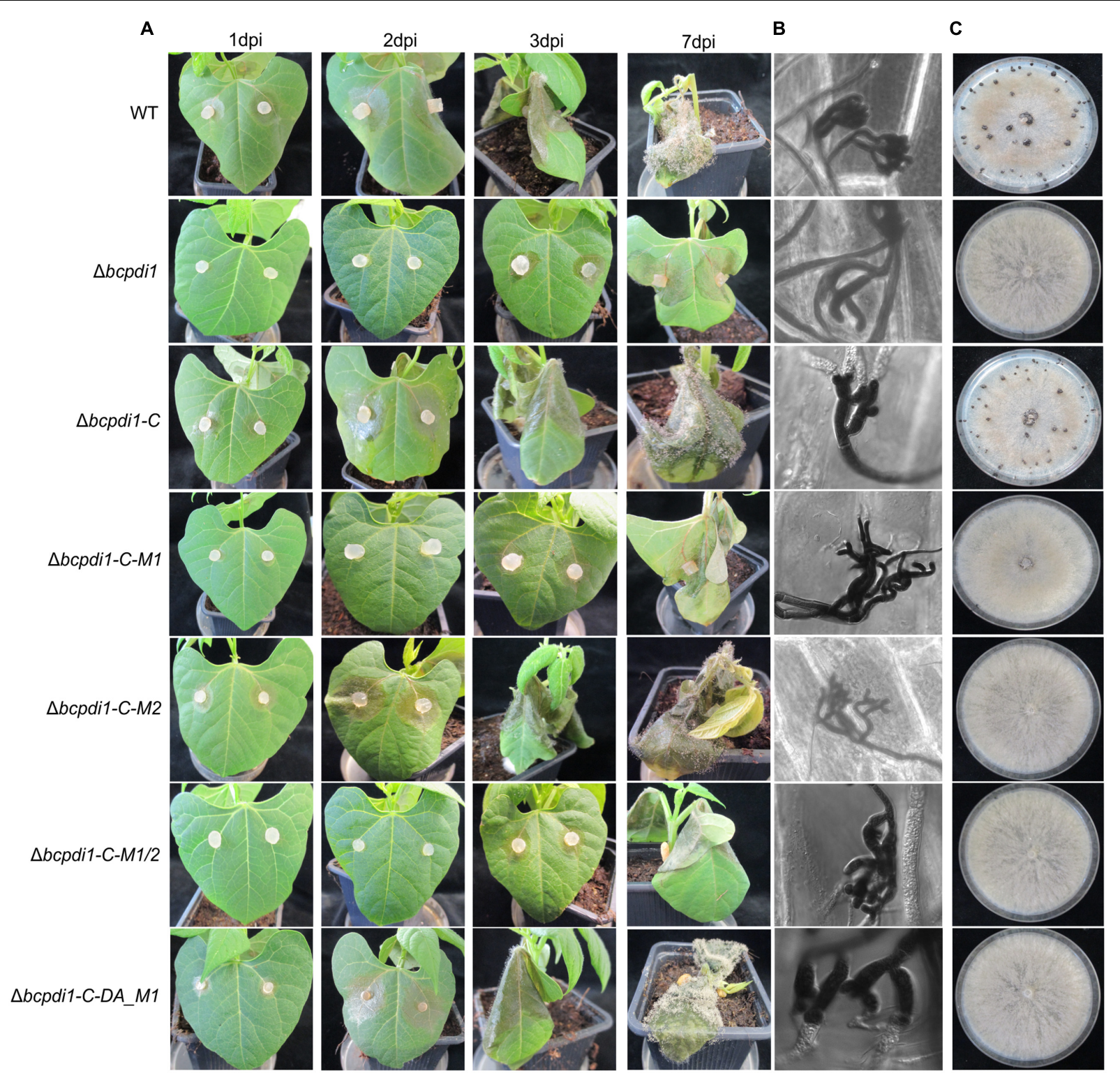

FIGURE 3 | The deletion of bcpdi1 influences mycelium mediated infection and the production of sclerotia. (A) Primary bean leaves were incubated with agar plugs from 3-day-old CM plates. Lesion diameters were measured and analyzed statistically. Biological replicates behaved similar. (B) The production of infection cushions was altered in mutants of bcpdi1. Agar plugs were incubated on onion epidermal layers for at least $20 \mathrm{~h}$. Lactophenol blue staining was accomplished to detect fungal material that is located on the surface of the onion layers. (C) BcPdi1 affects the production of sclerotia. CM Plates with agar plugs were incubated in constant darkness for at least 14 days. Biological replicates behaved similarly.

to be wild type like in both mutants. The Hacl homolog was upregulated in $\Delta b c p d i 1-C-D A \_r o G F P 2$ (Figure 5B). In summary, the results suggest that BcPdil is involved in the maintenance of the cytosolic redox state and may play a role in the UPR.

\section{BcPdi1 Interacts with Proteins of Nox-, $\mathrm{Ca}^{2+}$, and Redox Signaling Pathways}

To examine whether the overlapping functions between the Nox complex and PDI are based on physical/direct interaction of these proteins we used a yeast-two hybrid approach; since membrane associated proteins are involved, we chose the split-ubiquitin system which is optimized for monitoring interactions between transmembrane proteins. The protein BcPdil was shown to interact with the catalytic subunit of the NoxA complex, and to a minor extent with BcNoxB (Figure 6A and Supplementary Figure S2). The positive interaction of BcNoxA and BcPdil was confirmed in co-IP experiments (Figure 6B). Strains expressing BcNoxA.mcherry (lane 1) or BcPdil_GFP (lane 2) were used as controls (BcPdi1_GFP $=84$ kDa; BcNoxA_mcherry $=102 \mathrm{kDa}$ ) (see also Materials and Methods). Additional interactions were 

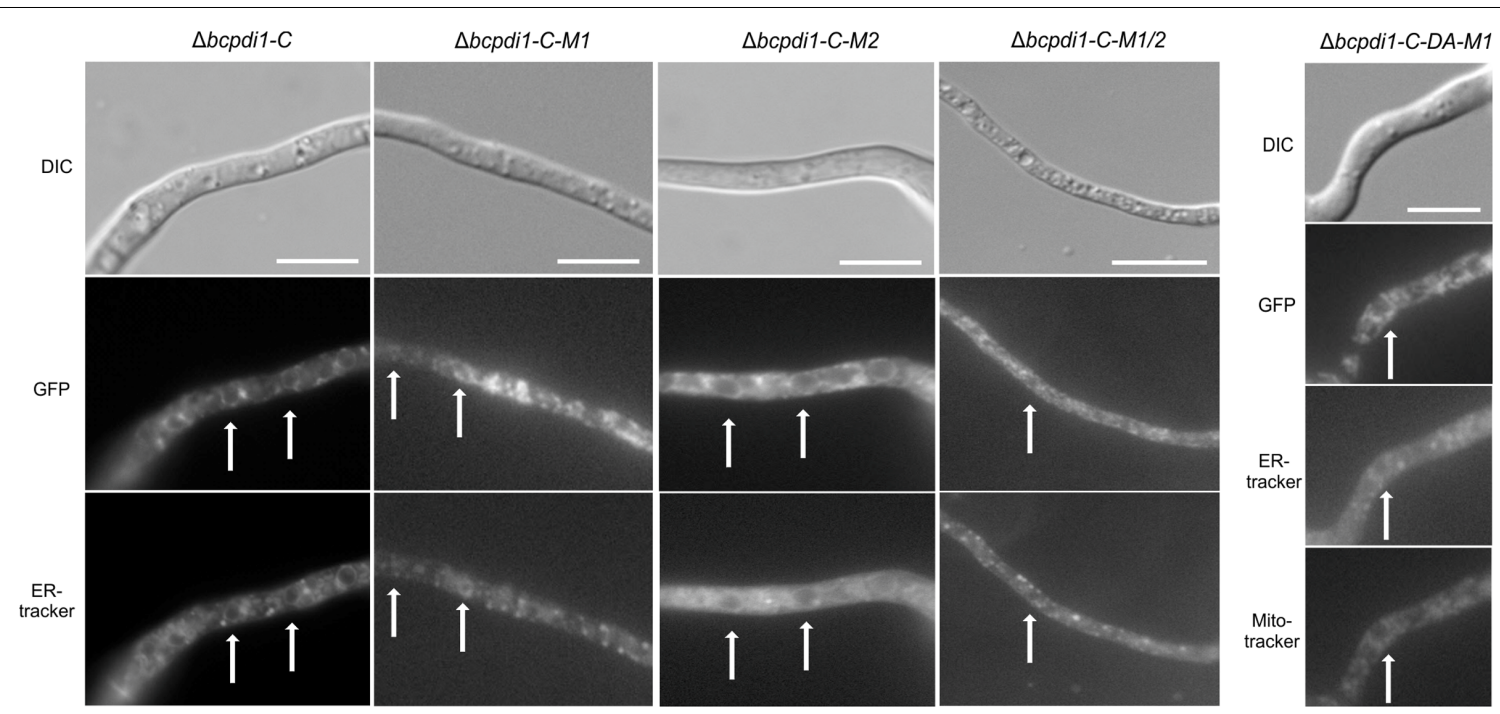

FIGURE 4 | BcPdi1 localizes to ER structures. Conidia of the strains expressing fusion constructs of BcPdi1 and GFP were cultivated overnight on microscopic slides in B5 medium supplemented with glucose (2\%). GFP fluorescence is visible in filamentous ER structures and inside the nuclear envelope. Dependent on the inserted mutation inside the BcPdi__GFP fusion construct (mimicking [de]phosphorylated) the fluorescence is shifted from the perinuclear localization to filamentous structures, which partially overlap with mitochondrial structures. Co-staining was performed with Hoechst 33342 and trackers for the detection of the ER (Blue-White DPX) or mitochondria (mitolDred). DIC, differential interference contrast microscopy.

shown for BcPdi1 with thioredoxin 2 (BcTrx2), an oxidoreductin (BcEro1) and the catalytic subunit of the calcineurin complex (BcCnA).

To elucidate the impact of the putative phosphorylation sites on the interaction with the different proteins, and also for the yeast-two hybrid based method, the putative cytosolic phosphorylation sites were mutated (BcPdil_M1 = T65A; BcPdi1_M2 = Y208A). Again, the first putative phosphorylation site was transferred into its putatively dominant active form (phospho mimic) (T65E).

Interaction tests were done comparatively with the mutated versions of BcPdil. While the mutation of the second predicted phosphorylation site (M2) had no effect on any of the interactions, mutation of M1 (T65A) and the phospho mimic led to the loss of the interaction with $\mathrm{BcCnA}$. There was no effect on the binding to NoxA/B, Trx2, and Ero1. (Figure 6 and Supplementary Figure S2).

To summarize these results it is clear that BcPdil interacts with members of various signaling cascades such as $\mathrm{Nox}_{-}^{-}, \mathrm{Ca}^{2+}$-, and redox related pathways. The catalytic subunit of calcineurin $(\mathrm{BcCnA})$ seems to bind to the cytosolic region of the protein and in particular to a segment exhibiting a single putative phosphorylation site.

In conclusion, we present evidence that BcPdil is involved in all major developmental processes, such as the formation of conidia, sclerotia and CATs. Moreover, the ER protein affects the virulence of the necrotrophic plant pathogen and mediates stress resistance to oxidative and osmotic stress. The phenotype of $\Delta b c p d i 1$ is similar to those of $\Delta b c n o x A$ and $\Delta b c n o x D$ mutants suggesting a link between Nox signaling and BcPdil in these differentiation processes. A cytosolic phosphorylation site, which is highly conserved among different species, clearly is essential for the function of BcPdil. In its phospho null form, the respective mutants behave like $\Delta b c p d i 1$. In contrast, the phospho mimic mostly restores the wild type phenotype.

\section{DISCUSSION}

Reactive molecules derived from oxygen and the redox homeostasis are essential parameters contributing to the complex lifestyle of the plant pathogen B. cinerea (Heller et al., 2012; Marschall and Tudzynski, 2016b; Marschall et al., 2016b). Especially during the interaction with its host, the fungus has to cope with external stimuli and needs to adapt itself to the changing environment. Major signals during the plant-pathogen interaction are ROS; these chemical species are produced by plants as a defense reaction, and also generated by the fungus during the intimate interaction with its host. In fungi, one of the main sources of the reactive molecules are NADPH oxidase (Nox) complexes that are transporting electrons across the lipid bilayer on to molecular oxygen (Bedard and Krause, 2007; Tudzynski et al., 2012). Although there has been tangible progress in the elucidation of the fungal Nox complexes in recent years, there are still many open questions that arise concerning Nox functionality, composition and the integration into existing signaling cascades.

Recent studies in $B$. cinerea revealed that Nox complexes have distinct functions within the ER (Siegmund et al., 2015; Marschall et al., 2016a). The ER is an important cellular organelle since essential processes such as protein processing (translation, folding, and transport), the UPR and the storage of metabolites take place in here. In fungi, information is scarce about the ER-proteins that are mediating these processes. 
A

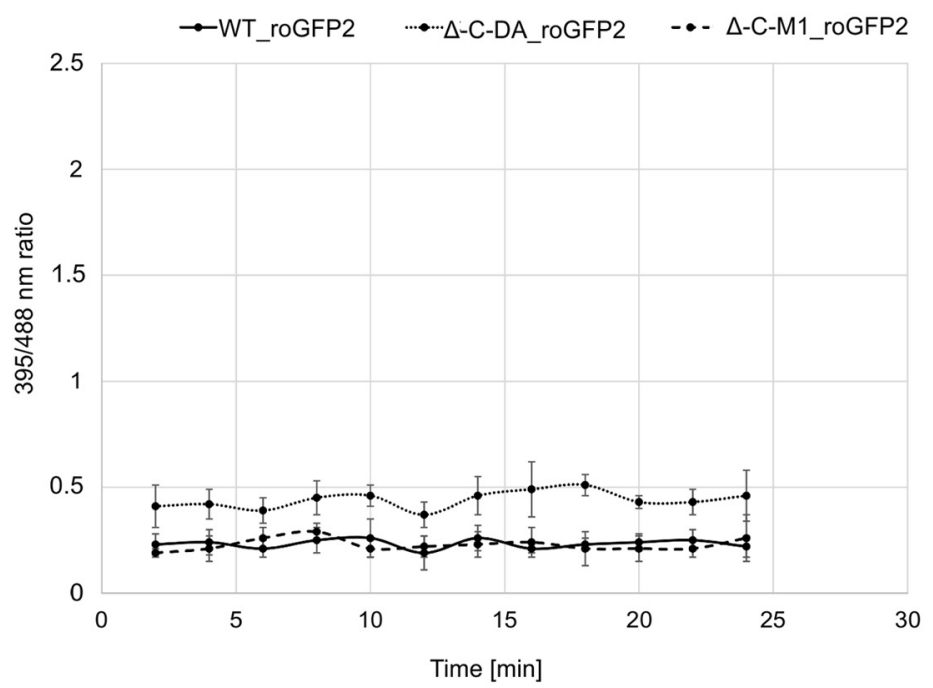

WT $\quad \Delta-\mathrm{C}-\mathrm{M} 1 \quad \Delta-\mathrm{C}-\mathrm{DA}$
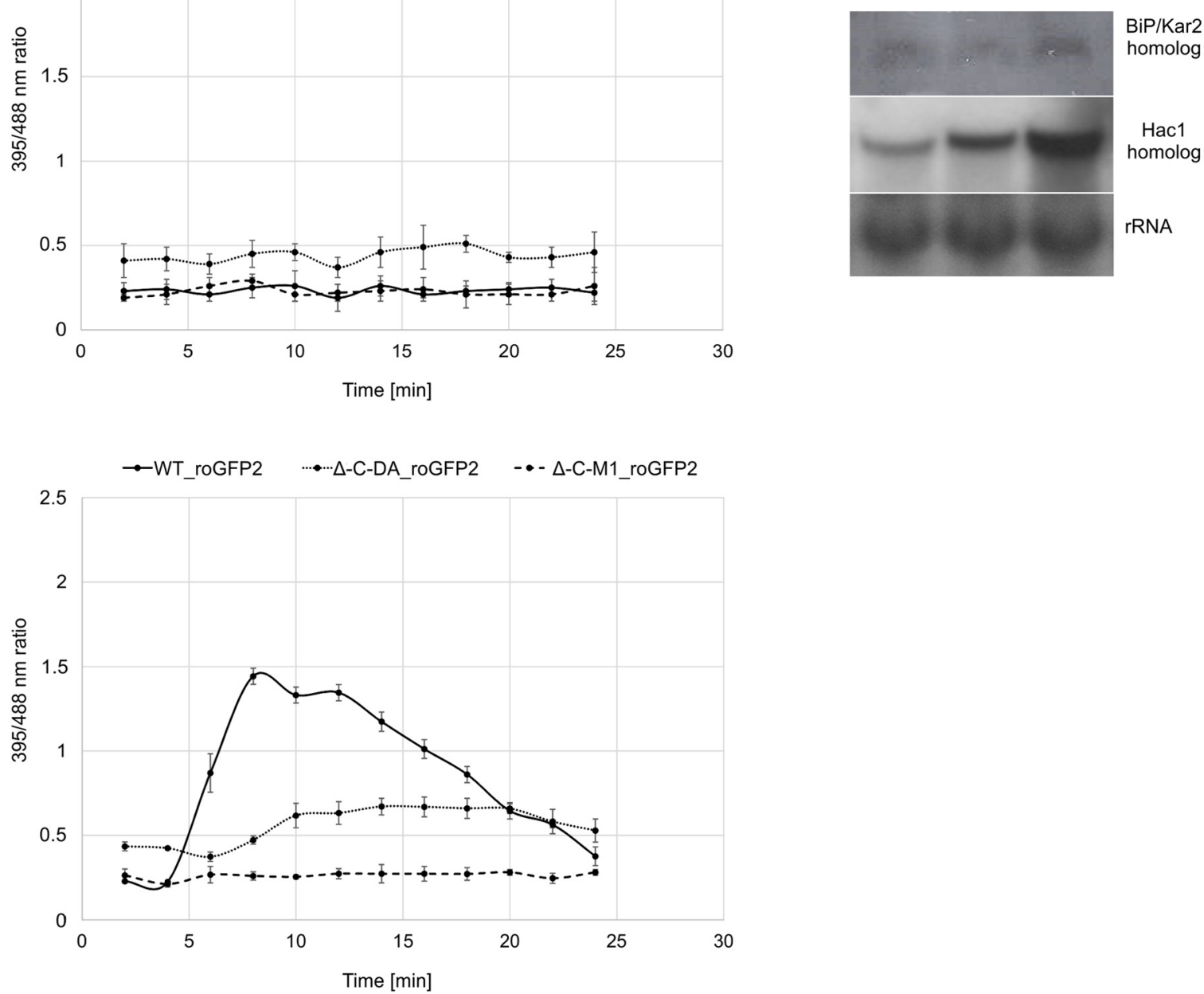

FIGURE 5 | Protein disulfide isomerase has an impact on the cytosolic redox state and the UPR. (A) Cytosolic roGFP2 constructs were transformed and expressed into $\Delta$-C-M1 and $\Delta$-C-DA. Z-stacks of images were taken by CLSM with excitation at 395 and $488 \mathrm{~nm}$. Ratio images were generated using the ImageJ software. The $395 / 488$ ratio inside the cytosol of the mutants was compared to the wild type under non-inducing conditions (above) and upon induction with $50 \mathrm{mM} \mathrm{CaCl} 2$ (below) after $4 \mathrm{~min}$. Mean values and standard deviations were calculated from five biological replicates. (B) Expression analysis of BiP/Kar2 and Hac1 homologs in the wild type and the mutants $\triangle b c p d i 1-C-M 1 \_r o G F P 2 / \triangle b c p d i 1-C-D A \_r o G F P 2$ via Northern blot studies. Strains were grown on CM medium overlayed with Cellophane for 3 days. RNA was isolated and expression was determined by Northern blotting. The rRNA probe was used as loading control.

In this study, we identified the ER protein BcPdil as new interaction partner of BcNoxA. In yeast-two hybrid experiments, as well as by co-immunoprecipitation, the ER protein was found to directly bind to the catalytic subunit of the NoxA complex. In other fungi, PDI is essential for viability (Farquhar et al., 1991) and is activated during ER-stress exposure, as in Trichoderma reesei (Saloheimo et al., 1999). Moreover, PDI is involved in the oxidative protein-folding machinery, which facilitates the formation of disulfide bonds (especially of secretory proteins) and the refolding of misfolded polypeptides. A direct interaction of BcPdil and BcNoxA might occur during the abovementioned folding process dependent on the ER redox state and putative co-factors (Mezghrani et al., 2001). A more likely hypothesis is that the interaction of both proteins is dependent on certain differentiation processes, as described in mammalian cultures in which Nox activity was shown to be dependent on PDI (Laurindo et al., 2008, reviewed in Zeeshan et al., 2016). In B. cinerea, putative interaction sites are the ER-located loops of the transmembrane protein 
A

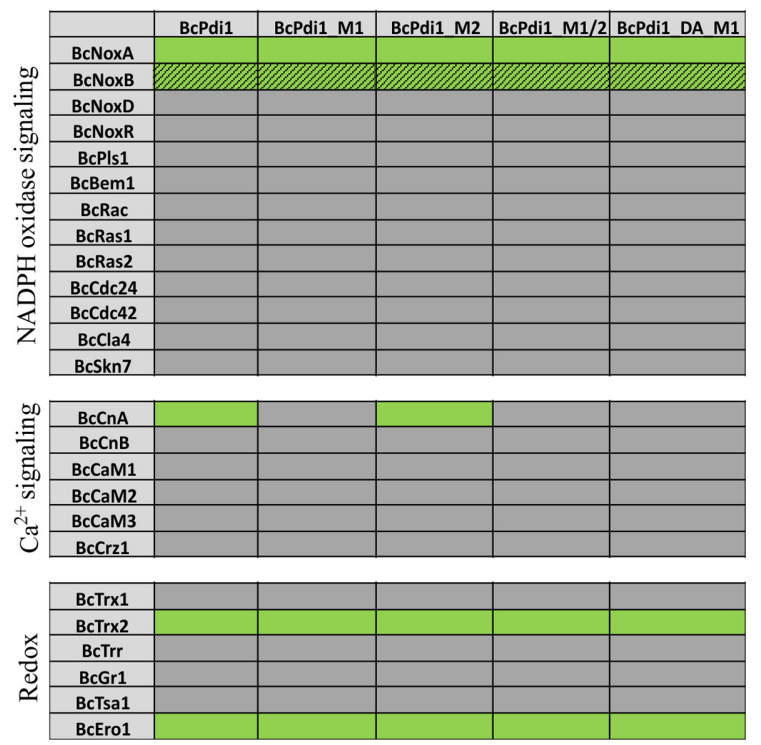

B

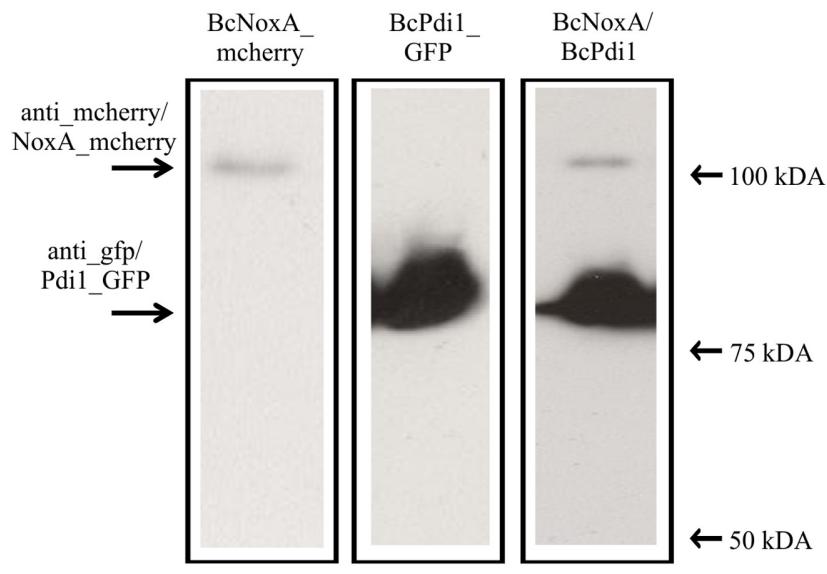

FIGURE 6 | PDI interacts with proteins of different signaling pathways. (A) In yeast-two hybrid assays, the interaction of BcPdi1 with proteins of different signaling cascades was analyzed. Besides the unmutated version, isoforms were included with mimicked dephosphorylated phosphorylation sites (M1/M2/M1_2) or the mimicked phosphorylated site (PDI_DA_M1). All constructs with the respective bait/prey protein were transformed in S. cerevisiae NMY51 and plated/dropped on selective media (SD- leu ${ }^{-}$his $^{-}$trp $^{-}$ade + Xgal +5 mM 3AT). If proteins interact, a growth on selective media will be possible. Positive interactions are illustrated in green, whereas lacing interactions were highlighted in gray. Streaky fields (gray/green coloration) indicate weak interactions. As negative controls, the respective empty prey/bait vector was co-transformed with the protein of interest. (B) BcNoxA and BcPdi1 interact in co-IP assays. For co-IP assays the strains expressing either BcNoxA_mcherry or BcPdi1_GFP were used as control strains. Interactions were analyzed with the strain B05.10 expressing both fusion constructs. After preparing the total protein extract, purification was achieved via $\mu$ MACS GFP beads/columns. All extracts were separated by Western blotting (from left to right: B05.10 + NoxA_mcherry; B05.10 + BcPdi1_GFP; B05.10 + BcNoxA_mcherry and BcPdi1_GFP). Anti-GFP or anti-mcherry antibodies were used for detection. In Lane 3 both fusion proteins were labeled with both antibodies simultaneously (BcPdi1_GFP = 84 kDa; BcNoxA_mcherry = 102 kDa).

BcNoxA, but it remains unclear in which direction the interaction between both proteins takes place in fungi. It is possible that $\mathrm{BcNoxA}$ works downstream of BcPdil since the phenotype of $\Delta b c p d i 1$ appeared to be more severely affected than the one of $\triangle b c n o x A$. Therefore, the binding of BcPdil could regulate $\mathrm{BcNoxA}$ activity in an manner still unknown, and perhaps similar to the mammalian system (Laurindo et al., 2008). In contrast, BcNoxA might work upstream of BcPdil and could be responsible for the transfer of electrons from NADPH to BcPdil. As a third possibility, the NoxA complex may actively produce superoxide, which is detoxified immediately by BcPdi1. Generation of superoxide would have the consequence that BcPdil would be shifted to its oxidized form, increasing its activity and thus allowing it to process a higher level of secretory proteins. This hypothesis is supported by findings that the levels of secreted proteins were altered in deletion mutants of bcnoxA and bcpdi1 (data not shown).

Nevertheless, the obtained data support the hypothesis that both BcNoxA and BcPdil are acting in the same signaling pathway(s). Consequently, all major developmental processes are affected in a similar way. Besides the impact on ICs, conidiospore production and formation of CATs, both proteins impact on virulence and formation of sclerotia. The more severe impact on virulence of $\Delta b c p d i 1$ may be attributed to the predicted two catalytic active domains located inside and outside of the ER. Therefore, the protein may influence signaling components which are located inside the ER, such as BcTrx2 (Viefhues et al., 2014), at the ER membrane, such as BcNoxA (Segmueller et al., 2008), as well as cytosolic components, such as BcCnA (Figure 6) (Harren et al., 2012). Since deletion mutants of bctrx2 appeared to be wild type like (Viefhues et al., 2014), the observed defects in vegetative and pathogenic processes are not attributable to this interaction. In contrast, the close association of BcPdil with the catalytic subunit of the phosphatase BcCNA might be more important. In former studies in our laboratory, mutants lacking bccna were generated. They displayed severe growth defects and appeared to be avirulent (Harren et al., 2012). To unravel which protein works upstream/downstream of each other and to elucidate the binding mechanism between BcPdil and BcCnA, the PDI was analyzed bioinformatically. Surprisingly, only two phosphorylation sites were predicted in the cytosolic loop of BcPdil. These sites may be putative target sites of regulating kinases and phosphatases. For both sites, mutants were generated mimicking the (de)phosphorylated version of the phosphorylation sites. Characterization revealed that the second predicted phosphorylation site (P-site) has no impact on binding to any interaction partner and had hardly any effect on growth and pathogenicity of the fungus (Figures 1-3). In contrast, the first putative P-site appeared 
to be essential for binding to the phosphatase and for every observed phenotype that had previously been described for the deletion mutant. It is noteworthy that both approaches, mimicking the phosphorylated and dephosphorylated version of the predicted P-site, are unable to restore growth typical of the wild type. Whereas the phospho null version resembles the phenotype of the deletion mutant, the phospho mimic version restored the wild type phenotype only partially. Thus, the results indicate that phosphorylation of $\mathrm{BcPdil}$ at the first predicted P-site is essential for its function, but it is remarkable that mimicking the phosphorylation does not have the opposite effect. The mutants displayed an intermediate phenotype between the deletion mutant (lack of CATs, sclerotia) and the wild type (stress sensitivity, virulence, and conidia production). This intermediate effect may be because the phosphorylation is only mimicked by the replacement of threonine by glutamic acid. Two indications that there may be even more complexity behind the phenotype was the localization of the protein and the cytosolic redox state of the PDI mutants. The protein localization of the BcPdi1_GFP fusion construct was monitored in the nuclear envelope as well as in filamentous structures of the ER (Figure 4). Whereas the mutation of the second putative P-site had no influence on the localization pattern, in the mutants mimicking the phosphorylation of the first predicted P-site the protein was localized in small filamentous structures and hardly detectable in the nuclear envelope. Costaining with ER- and mito-tracker agents revealed that both compartments seem to overlap. This phenomenon has previously been observed in mammalian cells in which the formation of tight mitochondria-ER complexes was dependent on stress conditions and involved in the regulation of the redox homeostasis and cell death (Kornmann and Walter, 2010; Grimm, 2012; Marchi et al., 2014; Stoica et al., 2014). To verify the presence of similar processes in $B$. cinerea, we analyzed the cytosolic redox state in the wild type in comparison to all mutants. While the unstimulated redox levels in $\Delta b c p d i 1$ (data not shown) and $\Delta b c p d i 1-\mathrm{C}-\mathrm{M} 1$ appeared to be as in the wild type, the mutants mimicking the phosphorylation of the first predicted P-site exhibited a higher $395 / 488$ ratio inside the cytosol. This unbalanced redox equilibrium became even more obvious under $\mathrm{Ca}^{2+}$ inducing conditions. Here in the wild type an oxidative burst is detectable upon $\mathrm{Ca}^{2+}$ stimulation. In previous studies, we were able to show that both Nox complexes contribute to the changes in redox status upon $\mathrm{Ca}^{2+}$ exposure. Surprisingly, the deletion mutant and $\Delta b c p d i 1-\mathrm{C}-\mathrm{M} 1$ also seem to be insensitive to the addition of $\mathrm{CaCl}_{2}$, suggesting that $\mathrm{BcPdi1}$ is part of the linear signal messaging of $\mathrm{Ca}^{2+}$ stress in close association to $\mathrm{BcNoxA}$ and $\mathrm{BcNoxB}$. All mutants mimicking the phosphorylation of the first putative $\mathrm{P}$-site displayed a very different pattern. A much higher 395/488 ratio and the absence of a wild type-like oxidative burst were monitored. To prove which cellular processes might contribute to this elevated 395/488 ratio, the expression of key genes of the UPR were analyzed (Graf et al., 2008; Jung et al., 2013; Heimel et al., 2013; Montenegro-Montero et al., 2015). Northern blot studies confirmed that the expression of the homolog to $\mathrm{Hacl}$ was significantly upregulated in the mutants mimicking the phosphorylation of the first predicted P-site (Figure 5). Therefore, the mutation of the first putative P-site may hinder the function of the PDI so that the redox homeostasis is unbalanced and rescue programs, such as the UPR, might be induced.

In summary, we show in this work that BcPdil is an ER protein, which is involved in several growth and differentiation processes such as formation of CATs, ICs, conidia, sclerotia as well as in virulence of $B$. cinerea. The phenotype of the deletion mutant resembles the ones of $\triangle b c n o x A$ and $\triangle b c n o x D$ and display - together with the physical interaction of BcNoxA and BcPdil - a strong link between ROS signaling and ER proteins and processes. The evidence at present is inconclusive as to which direction certain signals were forwarded in the pathways. Starting points for further studies might be the different tiepoints between ROS, the ER and $\mathrm{Ca}^{2+}$. Therefore, not only does the physical interaction of $\mathrm{BcPdil}$ and $\mathrm{BcCnA}$ hint to a rather close association, but even more to the collaboration of BcNoxA, BcNoxB and BcPdil upon exposure to $\mathrm{Ca}^{2+}$. Future studies should investigate how $\mathrm{Ca}^{2+}$ affects the regulation of Nox complexes, ER proteins and the redox homeostasis.

\section{AUTHOR CONTRIBUTIONS}

RM: conception, methodology, writing of the manuscript and realization of all experiments. PT: conception, review and editing. Final approval: RM and PT.

\section{FUNDING}

This work was funded by the DFG (Tu50/19).

\section{ACKNOWLEDGMENTS}

We thank Bettina Richter for excellent technical assistance, Kim Cohrs and Kai Heimel for discussion as well as Brian Williamson for critical reading of the manuscript.

\section{SUPPLEMENTARY MATERIAL}

The Supplementary Material for this article can be found online at: http://journal.frontiersin.org/article/10.3389/fmicb.2017. 00960/full\#supplementary-material

FIGURE S1 | Bioinformatics and bcpdi1 deletion strategy. (A) Multiple sequence alignment of BcPdi1 homologs of "Botrytis cinerea," "Aspergillus niger," "Neurospora crassa," and "Sclerotinia sclerotiorum." Alignment was performed by using MegAlign (DNAStar - Lasergene software) and the ClustalW alignment logarithm. Red boxes indicate important sites inside the sequence, such as phosphorylation sites (red arrows), catalytic sites (CXXC motif) or the putative calcineurin binding motif (box 2). The protein is predicted to be a transmembrane protein with two transmembrane domains, a small cytosolic and a large ER loop. Prediction was performed with the programs TMHMM (http://www.cbs.dtu.dk/services/TMHMM/) and SACS MEMSAT2 (Jones et al., 1994). Phosphorylation sites were predicted by KinasePhos (Huang et al., 2005). (B) Diagnostic PCR shows the deletion of bcpdi1 using the primers: 5' 
homologous integration $32+43,3^{\prime}$ homologous integration: $33+42, \mathrm{WT}$ : $23+24$ (see Supplementary Table S2). (C) Southern blot analysis of three independent $\Delta$ bcpdi1 mutants illustrates the absence of any ectopic integrations of the deletion cassette. Genomic DNA was digested with Pvul; detection took place with the $5^{\prime}$ flank (dotted line). For the WT a fragment size of $5.2 \mathrm{~kb}$ was expected while the fragments of the mutants are expected at a size of $3.5 \mathrm{~kb}$. The bcpdi1 gene was replaced by a hygromycin resistance cassette by homologous recombination via the $5^{\prime}$ and $3^{\prime}$ flank of the gene.

FIGURE S2 | BcPdi1 interacts with different proteins of various signaling cascades in yeast-two hybrid assays. Proteins of interest were co-transformed in to

\section{REFERENCES}

Armentrout, V. M., and Downer, A. J. (1987). Infection cushion development by Rhizoctonia solarti on cotton. Phytopath 77:619. doi: 10.1094/Phyto77-619

Baksh, S., Burns, K., Andrin, C., and Michalak, M. (1995). Interaction of calreticulin with protein disulfide isomerase. J. Biol. Chem. 270, 31338-31344. doi: $10.1074 /$ jbc. 270.52 .31338

Beckman, K. B., and Ames, B. N. (1998). The free radical theory of aging matures. Physiol. Rev. 78, 547-581.

Bedard, K., and Krause, K. H. (2007). The NOX family of ROS-generating NADPH oxidases: physiology and pathophysiology. Physiol. Rev. 87, 245-313. doi: 10. 1152/physrev.00044.2005

Broome, J. C., English, J. T., Marois, J. J., Latorre, B. A., and Aviles, J. C. (1995). Development of an infection model for Botrytis bunch rot of grapes based on wetness duration and temperature. Phytopathology 85, 97-102. doi: 10.1094/ Phyto-85-97

Buettner, P., Koch, F., Voigt, K., Quidde, T., Risch, S., Blaich, R., et al. (1994). Variations in ploidy among isolates of Botrytis cinerea: implications for genetic and molecular analyses. Curr. Genet. 25, 445-450. doi: 10.1007/BF00 351784

Cenis, J. L. (1992). Rapid extraction of fungal DNA for PCR amplification. Nucleic Acids Res. 20:2380. doi: 10.1093/nar/20.9.2380

Church, G. M., and Gilbert, W. (1984). Genomic sequencing. Proc. Natl. Acad. Sci. U.S.A. 81, 1991-1995. doi: 10.1073/pnas.81.7.1991

Colot, H. V., Park, G., Turner, G. E., Ringelberg, C., Crew, C. M., Litvinkova, L., et al. (2006). A high-throughput gene knockout procedure for Neurospora reveals functions for multiple transcription factors. Proc. Natl. Acad. Sci. U.S.A. 103, 10352-10357. doi: 10.1073/pnas.0601456103

Corbett, E. F., and Michalak, M. (2000). Calcium, a signaling molecule in the endoplasmic reticulum? Trends Biochem. Sci. 25, 307-311.

de A Paes, A. M., Verissimo-Filho, S., Guimaraes, L. L., Silva, A. C., Takiuti, J. T., Santos, C. X., et al. (2011). Protein disulfide isomerase redox-dependent association with $\mathrm{p} 47$ (phox): evidence for an organizer role in leukocyte NADPH oxidase activation. J. Leukoc. Biol. 90, 799-810. doi: 10.1189/jlb. 0610324

Doehlemann, G., Berndt, P., and Hahn, M. (2006). Different signalling pathways involving a $\mathrm{G} \alpha$ protein, cAMP and a MAP kinase control germination of Botrytis cinerea conidia. Mol. Microbiol. 59, 821-835. doi: 10.1111/j.1365-2958. 2005.04991.x

Elad, Y., Pertot, I., Cores-Prado, A. M., and Stewart, A. (2016). "Plant hosts of Botrytis spp," in Botrytis - The Fungus, The Pathogen and its Management in Agricultural Systems, eds S. Fillinger and Y. Elad (Berlin: Springer).

Farquhar, R., Honey, N., Murant, S. J., Bossier, P., Schultz, L., Montgomery, D., et al. (1991). Protein disulfide isomerase is essential for viability in Saccharomyces cerevisiae. Gene 108, 81-89. doi: 10.1016/0378-1119(91)90490-3

Fernandes, D. C., Manoel, A. H. O., Wosniak, J., and Laurindo, F. R. (2009). Protein disulfide isomerase overexpression in vascular smooth muscle cells induces spontaneous preemptive NADPH oxidase activation and Nox1 mRNA expression: effects of nitrosothiol exposure. Arch. Biochem. 484, 197-204. doi: 10.1016/j.abb.2009.01.022

Gietz, R. D., and Woods, R. A. (2002). Transformation of yeast by lithium acetate/single-stranded carrier DNA/polyethylene glycol method. Methods Enzymol. 350, 87-96. doi: 10.1016/S0076-6879(02)50957-5
S. cerevisiae NMY51 and plated on selective media (SD-leu ${ }^{-} \mathrm{trp}^{-} /$ $\left(\mathrm{SD}^{-}\right.$leu ${ }^{-}$his $^{-}$trp $^{-}$ade). For drop tests, the yeast strains were cultivated in YPAD medium, starved out in $1 \mathrm{M}$ sorbitol for 5-6 $\mathrm{h}$ and dropped on to selective media

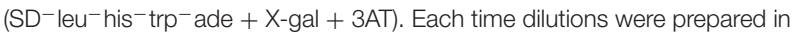
advance (1:10 up to 1:1000). For negative controls, the respective empty vector was co-transformed. For the positive control, the vecto constructs POST_Nubl was co-transformed.

TABLE S1 | Strains used in this work.

TABLE S2 | Oligonucleotides used in this work.

Gomi, M., Akazawa, F., and Mitaku, S. (2000). SOSUIsignal: software system for prediction of signal peptide and membrane protein. Genome Inform. 11, 414-415.

Govrin, E. M., and Levine, A. (2000). The hypersensitive response facilitates plant infection by the necrotrophic pathogen Botrytis cinerea. Curr. Biol. 10, 751-757. doi: 10.1016/S0960-9822(00)00560-1

Graf, A., Gasser, B., Dragosits, M., Sauer, M., Leparc, G. G., Tüchler, T., et al. (2008). Novel insights into the unfolded protein response using Pichia pastoris specific DNA microarrays. BMC Genom. 9:390. doi: 10.1186/1471-2164-9-390

Grimm, S. (2012). The ER-mitochondria interface: the social network of cell death. Biochim. Biophys. Acta (BBA)-Mol. Cell Res. 1823, 327-334. doi: 10.1016/j. bbamcr.2011.11.018

Gronover, C. S., Kasulke, D., Tudzynski, P., and Tudzynski, B. (2001). The role of G protein alpha subunits in the infection process of the gray mold fungus Botrytis cinerea. Mol. Plant Microbe. Interact. 11, 1293-1302. doi: 10.1094/MPMI.2001. 14.11.1293

Haefliger, S., Klebig, C., Schaubitzer, K., Schardt, J., Timchenko, N., Mueller, B. U., et al. (2011). Protein disulfide isomerase blocks CEBPA translation and is upregulated during the unfolded protein response in AML. Blood 117, 5931-5940. doi: 10.1182/blood-2010-08-304485

Harren, K., Schumacher, J., and Tudzynski, B. (2012). The $\mathrm{Ca}^{2+} /$ calcineurindependent signaling pathway in the gray mold Botrytis cinerea: the role of calcipressin in modulating calcineurin activity. PLoS ONE 7:e41761. doi: 10 . 1371/journal.pone.0041761

Heimel, K., Freitag, J., Hampel, M., Ast, J., Bolker, M., and Kamper, J. (2013). Crosstalk between the unfolded protein response and pathways that regulate pathogenic development in Ustilago maydis. Plant Cell 25, 4262-4277. doi: 10.1105/tpc.113.115899

Heller, J., Meyer, A. J., and Tudzynski, P. (2012). Redox-sensitive GFP2: use of the genetically encoded biosensor of the redox status in the filamentous fungus Botrytis cinerea. Mol. Plant Pathol. 8, 935-947. doi: 10.1111/j.1364-3703.2012. 00802.x

Heller, J., and Tudzynski, P. (2011). Reactive oxygen species in phytopathogenic fungi: signaling, development, and disease. Annu. Rev. Phytopathol. 49, 369390. doi: 10.1146/annurev-phyto-072910-095355

Huang, H. D., Lee, T. Y., Tzeng, S. W., and Horng, J. T. (2005). KinasePhos: a web tool for identifying protein kinase-specific phosphorylation sites. Nucleic Acids Res. 33, W226-W229. doi: 10.1093/nar/gki471

Janiszewski, M., Lopes, L. R., Carmo, A. O., Pedro, M. A., Brandes, R. P., Santos, C. X., et al. (2005). Regulation of $\mathrm{NAD}(\mathrm{P}) \mathrm{H}$ oxidase by associated protein disulfide isomerase in vascular smooth muscle cells. J. Biol. Chem. 280, 4081340819. doi: 10.1074/jbc.M509255200

Jones, D. T., Taylor, W. R., and Thornton, J. M. (1994). A model recognition approach to the prediction of all-helical membrane protein structure and topology. Biochemistry 33, 3038-3049. doi: 10.1021/bi00176a037

Jung, K.-W., Kang, H. A., and Bahn, Y.-S. (2013). Essential roles of the Kar2/BiP molecular chaperone downstream of the UPR pathway in Cryptococcus neoformans. PloS ONE 8:e58956. doi: 10.1371/journal.pone.0058956

Kersteen, E. A., and Raines, R. T. (2003). Catalysis of protein folding by protein disulfide isomerase and small-molecule mimics. Antioxid. Redox Signal. 5, 413-424. doi: 10.1089/152308603768295159

Kim, J., and Mayfield, S. P. (1997). Protein disulfide isomerase as a regulator of chloroplast translational activation. Science (New York, N.Y.) 278, 1954-1957. doi: $10.1126 /$ science.278.5345.1954 
Klimpel, A., Gronover, C. S., Williamson, B., Stewart, J. A., and Tudzynski, B. (2002). The adenylate cyclase (BAC) in Botrytis cinerea is required for full pathogenicity. Mol. Plant Pathol. 3, 439-450. doi: 10.1046/j.1364-3703.2002. 00137.x

Ko, H. S., Uehara, T., and Nomura, Y. (2002). Role of ubiquilin associated with protein-disulfide isomerase in the endoplasmic reticulum in stressinduced apoptotic cell death. J. Biol. Chem. 277, 35386-35392. doi: 10.1074/jbc. M203412200

Koch, G. L. (1990). The endoplasmic reticulum and calcium storage. Bioessays 12, 527-531. doi: 10.1002/bies.950121105

Kornmann, B., and Walter, P. (2010). ERMES-mediated ER-mitochondria contacts: molecular hubs for the regulation of mitochondrial biology. J. Cell Sci. 123(Pt 9), 1389-1393. doi: 10.1242/jcs.058636

Lambeth, J. D. (2004). NOX enzymes and the biology of reactive oxygen. Nat. Rev. Immunol. 4, 181-189. doi: 10.1038/nri1312

Laurindo, F. R., Araujo, T. L., and Abrahao, T. B. (2014). Nox NADPH oxidases and the endoplasmic reticulum. Antioxid. Redox Signal. 20, 2755-2775. doi: 10.1089/ars.2013.5605

Laurindo, F. R., Fernandes, D. C., Amanso, A. M., Lopes, L. R., and Santos, C. X. C. (2008). Novel role of protein disulfide isomerase in the regulation of NADPH oxidase activity: pathophysiological implications in vascular diseases. Antioxid. Redox Signal. 10, 1101-1114. doi: 10.1089/ars.2007.2011

Li, C. P., and Larkins, B. A. (1996). Expression of protein disulfide isomerase is elevated in the endosperm of the maize floury-2 mutant. Plant Mol. Biol. 30, 873-882. doi: 10.1007/BF00020800

Lucero, H. A., Lebeche, D., and Kaminer, B. (1998). ER calcistorin/protein-disulfide isomerase acts as a calcium storage protein in the endoplasmic reticulum of a living cell. Comparison with calreticulin and calsequestrin. J. Biol. Chem. 273, 9857-9863. doi: 10.1074/jbc.273.16.9857

Madamanchi, N. R., and Runge, M. S. (2010). NADPH oxidases and atherosclerosis: unraveling the details. Am. J. Physiol. Heart Circ. Physiol. 298, H1-H2. doi: 10.1152/ajpheart.01020.2009

Marchi, S., Patergnani, S., and Pinton, P. (2014). The endoplasmic reticulummitochondria connection: one touch, multiple functions. Biochim. Biophys. Acta (BBA) 1837, 461-469. doi: 10.1016/j.bbabio.2013.10.015

Marschall, R., Siegmund, U., Burbank, J., and Tudzynski, P. (2016a). Update on Nox function, site of action and regulation in Botrytis cinerea. Fungal Biol. Biotechnol. 3:8. doi: 10.1186/s40694-016-0026-6

Marschall, R., Schumacher, J., Siegmund, U., and Tudzynski, P. (2016b). Chasing stress signals - exposure to extracellular stimuli differentially affects the redox state of cell compartments in the wild type and signaling mutants of Botrytis cinerea. Fung. Genet. Biol. 90, 12-22. doi: 10.1016/j.fgb.2016.03.002

Marschall, R., and Tudzynski, P. (2016a). BcIqg1, a fungal IQGAP homolog, interacts with NADPH oxidase, MAP kinase and calcium signaling proteins and regulates virulence and development in Botrytis cinerea. Mol. Microbiol. 101, 281-298. doi: 10.1111/mmi.13391

Marschall, R., and Tudzynski, P. (2016b). Reactive oxygen species in development and infection processes. Sem. Cell and Dev. Biol. 57, 138-146. doi: 10.1016/j. semcdb.2016.03.020

McIlvaine, T. C. (1921). A buffer solution for colorimetric comparison. J. Biol Chem. 49, 183-186.

Merksamer, P. I., Trusina, A., and Papa, F. R. (2008). Real-time redox measurements during endoplasmic reticulum stress reveal interlinked protein folding functions. Cell 135, 933-947. doi: 10.1016/j.cell.2008.10.011

Mezghrani, A., Fassio, A., Benham, A., Simmen, T., Braakman, I., and Sitia, R. (2001). Manipulation of oxidative protein folding and PDI redox state in mammalian cells. EMBO J. 20, 6288-6296. doi: 10.1093/emboj/20.22.6288

Montenegro-Montero, A., Goity, A., and Larrondo, L. F. (2015). The bZIP transcription factor HAC-1 is involved in the unfolded protein response and is necessary for growth on cellulose in Neurospora crassa. PLoS ONE 10:e0131415. doi: 10.1371/journal.pone.0131415

Mori, K., Ogawa, N., Kawahara, T., Yanagi, H., and Yura, T. (1998). Palindrome with spacer of one nucleotide is characteristic of the cis-acting unfolded protein response element in Saccharomyces cerevisiae. J. Biol. Chem. 273, 9912-9920. doi: $10.1074 / j b c .273 .16 .9912$

Mori, K., Sant, A., Kohno, K., Normington, K., Gething, M. J., and Sambrook, J. F. (1992). A 22 bp cis-acting element is necessary and sufficient for the induction of the yeast KAR2 (BiP) gene by unfolded proteins. EMBO J. 11, 2583-2593.
Ngiam, C., Jeenes, D. J., Punt, P. J., Van Den Hondel, C. A., and Archer, D. B. (2000). Characterization of a foldase, protein disulfide isomerase $\mathrm{A}$, in the protein secretory pathway of Aspergillus niger. Appl. Environ. Microbiol. 66, 775-782. doi: 10.1128/AEM.66.2.775-782.2000

Perri, E. R., Thomas, C. J., Parakh, S., Spencer, D. M., and Atkin, J. D. (2016). The unfolded protein response and the role of protein disulfide isomerase in neurodegeneration. Front. Cell Dev. Biol. 3:80. doi: 10.3389/fcell.2015.00080

Pontecorvo, G., Roper, J. A., Hemmons, L. M., MacDonald, K. D., and Bufton, A. W. (1953). The genetics of Aspergillus nidulans. Adv. Genet. 5, 141-238. doi: 10.1016/s0065-2660(08)60408-3

Roca, M. G., Weichert, M., Siegmund, U., Tudzynski, P., and Fleissner, A. (2012). Germling fusion via conidial anastomosis tubes in the grey mold Botrytis cinerea requires NADPH oxidase activity. Fungal Biol. 116, 379-387. doi: 10.1016/j. funbio.2011.12.007

Roy, K., Wu, Y., Meitzler, J. L., Juhasz, A., Liu, H., Jiang, G., et al. (2015). NADPH oxidases and cancer. Clin. Sci. 128, 863-875. doi: 10.1042/CS20140542

Saloheimo, M., Lund, M., and Penttilä, M. E. (1999). The protein disulphide isomerase gene of the fungus Trichoderma reesei is induced by endoplasmic reticulum stress and regulated by the carbon source. Mol. Gen. Genet. MGG 262, 35-45. doi: 10.1007/s004380051057

Schumacher, J. (2012). Tools for Botrytis cinerea: new expression vectors make the gray mold fungus more accessible to cell biology approaches. Fungal Genet. Biol. 49, 483-497. doi: 10.1016/j.fgb.2012.03.005

Schumacher, J., Pradier, J. M., Simon, A., Traeger, S., Moraga, J., Collado, I. G., et al. (2012). Natural variation in the VELVET gene bcvel1 affects virulence and light-dependent differentiation in Botrytis cinerea. PLoS ONE 7:e47840. doi: 10.1371/journal.pone.0047840

Schwarzlaender, M., Fricker, M. D., Mueller, C., Marty, L., Brach, T., Novak, J., et al. (2008). Confocal imaging of glutathione redox potential in living plant cells. J. Microsc. 231, 299-316. doi: 10.1111/j.1365-2818.2008. 02030.x

Segmueller, N., Kokkelink, L., Giesbert, S., Odinius, D., van Kan, J., and Tudzynski, P. (2008). NADPH oxidases are involved in differentiation and pathogenicity in Botrytis cinerea. Mol. Plant Microbe Interact. 21, 808-819. doi: 10.1094/MPMI-21-6-0808

Siegmund, U., Heller, J., van Kan, J. A., and Tudzynski, P. (2013). The NADPH oxidase complexes in Botrytis cinerea: evidence for a close association with the ER and the tetraspanin Pls1. PLoS ONE 8:e55879. doi: 10.1371/journal.pone. 0055879

Siegmund, U., Marschall, R., and Tudzynski, P. (2015). BcNoxD, a putative ER protein, is a new component of the NADPH oxidase complex in Botrytis cinerea. Mol. Microbiol. 95, 988-1005. doi: 10.1111/mmi.12869

Sievers, F., Wilm, A., Dineen, D., Gibson, T. J., Karplus, K., Li, W., et al. (2011). Fast, scalable generation of high-quality protein multiple sequence alignments using clustal omega. Mol. Syst. Biol. 7:539. doi: 10.1038/msb.2011.75

Steinhorst, L., and Kudla, J. (2014). Signaling in cells and organisms-calcium holds the line. Curr. Opin. Plant Biol. 22, 14-21. doi: 10.1016/j.pbi.2014. 08.003

Stoica, R., De Vos, K. J., Paillusson, S., Mueller, S., Sancho, R. M., Lau, K., et al. (2014). ER-mitochondria associations are regulated by the VAPB-PTPIP51 interaction and are disrupted by ALS/FTD-associated TDP-43. Nat. Commun. 5:3996. doi: $10.1038 /$ ncomms 4996

Sullivan, D. C., Huminiecki, L., Moore, J. W., Boyle, J. J., Poulsom, R., Creamer, D., et al. (2003). EndoPDI, a novel protein-disulfide isomerase-like protein that is preferentially expressed in endothelial cells acts as a stress survival factor. J. Biol. Chem. 278, 47079-47088. doi: 10.1074/jbc.M308124200

Tan, K. K., and Epton, H. (1973). Effect of light on the growth and sporulation of Botrytis cinerea. Trans. Br. Mycol. Soc. 61, 145-157. doi: 10.3389/fpls.2016. 00625

Tudzynski, P., Heller, J., and Siegmund, U. (2012). Reactive oxygen species generation in fungal development and pathogenesis. Curr. Opin. Microbiol. 15, 653-659. doi: 10.1016/j.mib.2012.10.002

Viefhues, A., Heller, J., Temme, N., and Tudzynski, P. (2014). Redox systems in Botrytis cinerea: impact on development and virulence. Mol. Plant-Microbe. Interact. 27, 858-874. doi: 10.1094/MPMI-01-14-0012-R

Wilkinson, B. L., and Landreth, G. E. (2006). The microglial NADPH oxidase complex as a source of oxidative stress in alzheimer's disease. J. Neuroinflammation 3:30. doi: 10.1186/1742-2094-3-30 
Zeeshan, H. M. A., Lee, G. H., Kim, H., and Chae, H. (2016). Endoplasmic reticulum stress and associated ROS. Int. J. Mol. Sci. 17:327. doi: 10.3390/ ijms 17030327

Conflict of Interest Statement: The authors declare that the research was conducted in the absence of any commercial or financial relationships that could be construed as a potential conflict of interest.
Copyright $\odot 2017$ Marschall and Tudzynski. This is an open-access article distributed under the terms of the Creative Commons Attribution License (CC BY). The use, distribution or reproduction in other forums is permitted, provided the original author(s) or licensor are credited and that the original publication in this journal is cited, in accordance with accepted academic practice. No use, distribution or reproduction is permitted which does not comply with these terms. 